\title{
Penetration of Ubiquinone (Q10) Nanoemulsion Using Olive Oil Through Rat Skin by Tristiana Erawati
}

Submission date: 01-Mar-2019 11:44AM (UTC+0800)

Submission ID: 1085774582

File name: ation_of_Ubiquinone_Q10_Nanoemulsion_Using_Olive_Oil_Through.pdf (720.84K) Word count : 2151 
Research Article

\title{
Penetration of Ubiquinone (Q10) Nanoemulsion Using Olive Oil Through Rat Skin
}

\author{
Deapsari F, Erawati T M*, Soeratri W \\ Pharmaceutics Department of Faculty of Pharmacy, Airlangga University \\ Kampus B UNAIR, Jl Dharmawangsa Dalam Surabaya, Indonesia
}

Available Online: $25^{\text {th }}$ February, 2017

\begin{abstract}
Ubiquinon (Q10) is an endogenous antioxidant. It is lipophilic and practically insoluble in water. To improve its solubility and penetration, it was formulated into $\mathrm{O} / \mathrm{W}$ nanoemulsion. The resulting Q10 nanoemulsion was then physically characterized for its droplet size, morphology, and viscosity. In addition, it was conducted a penetration test of Q10 in nanoemulsion delivery system compared to Q10 in emulsion using male Wistar rats. From the characterization results, it was found that the droplet size of Q10 nanoemulsion $(70.07 \pm 12.42 \mathrm{~nm})<$ Q10 emulsion $(21.063 \pm 3,57 \mu \mathrm{m})$, the morphology of droplet Q10 nanoemulsion and Q10 emulsion that are spherical, and that the viscosity of Q10 nanoemulsion (10.5 CPa.s) < Q10 emulsion (16 CPa.s). The penetration rate of Q10 in nanoemulsion and emulsion was observed at 2 hours, 4 hours, and 6 hours after treatment. The result was known that the penetration rate of Q10 in nanoemulsion $(174.49 \mu \mathrm{m} / \mathrm{h})>$ Q10 in emulsion $(20.429 \mu \mathrm{m} / \mathrm{h})$.
\end{abstract}

Keywords: nanoemulsion, olive oil, penetration, Q10.

\section{INTRODUCTION}

Ubiquinone (Q10) is an endogenous antioxidant. It has an important role for energy production in body cells, stimulates the body's immune system, increases tissue oxygenation, and has antiaging effects ${ }^{1}$. Q10 is lipophilic with a $\log P$ of 3.63 and a molecular weight of 863.36 $\mathrm{g} / \mathrm{mol}$, it a practically in soluble material. To improve its solubility and penetration, Q10 is formulated in an $\mathrm{O} / \mathrm{W}$ type nanoemulsion. The small droplet size of nanoemulsion will increase the penetration rate and can increase the effectiveness ${ }^{2}$

Nanoemulsion consists of four components, namely: oil, water, surfactants, and co-surfactants. In this research, olive oil is used as an oil phase which is known to be the one that has the highest content of fatty acids, namely palmitic acid, oleic acid, and linoleic acid. Palmitic acid is used as an enhancer and was known non-irritating, whereas oleic acid can improve the bioavailability of lipophilic drug substance, as an enhancer mechanism is to modify permeability of the stratum corneum ${ }^{3}$. Linoleic acid is used in the transdermal formulation as an emulsifiying agent ${ }^{3}$. When viewed from the fatty acid structure, the Q10 nanoemulsion with olive oil phase is likely to have a greater penetration, thereby increasing the effectiveness of Q10

\section{MATERIALS AND METHODS}

Q10 (Kangcare), olive oil (Chemical Biologia Ambiente), Tween 80 (Sigma), Span 80 (Sigma), and 96\% Ethanol (Merck). Acetate buffer $\mathrm{pH} 4.2 \pm 0.2$ is made of glacial acetic acid and sodium acetate (Merck) with pro-analysis quality.

Research Instruments

Thermo shaker, cone and plate viscometer, Transmission Electron Microscope (TEM) JEOL JEM 1400, Delsa ${ }^{\text {TM }}$ Submicron Nano Particle Size and Zeta Potential Dynamic Light Scattering, light microscope (Olympus), Microscope Flourecents and Brightfield Olympus FSX100.

Experimental Animals

Animals used were male Wistar rats with the inclusion criteria: 8-10 weeks of age, 100-250 grams of weight, healthy condition, with no defect or injury.

Nanoemulsion Preparation

The nanoemulsion formula in this study refers to the modified formula from Erawati et al, 2014. ${ }^{4}$ In this experiment the concentration of oils, surfactants, and

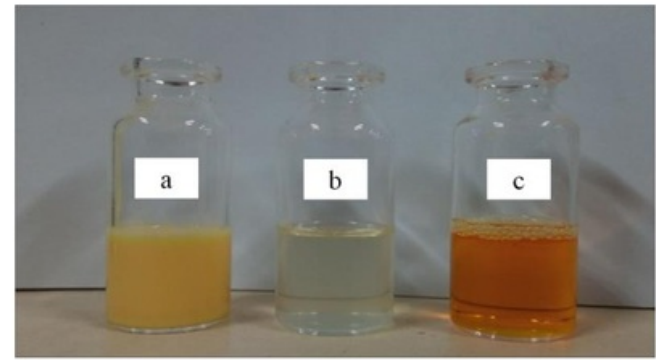

Figure 1: The preparation of Q10 nanoemulsion (c), blank nanoemulsion (b), and Q10 emulsion (a)

*Author for Correspondence: era ffua@yahoo.co.id 


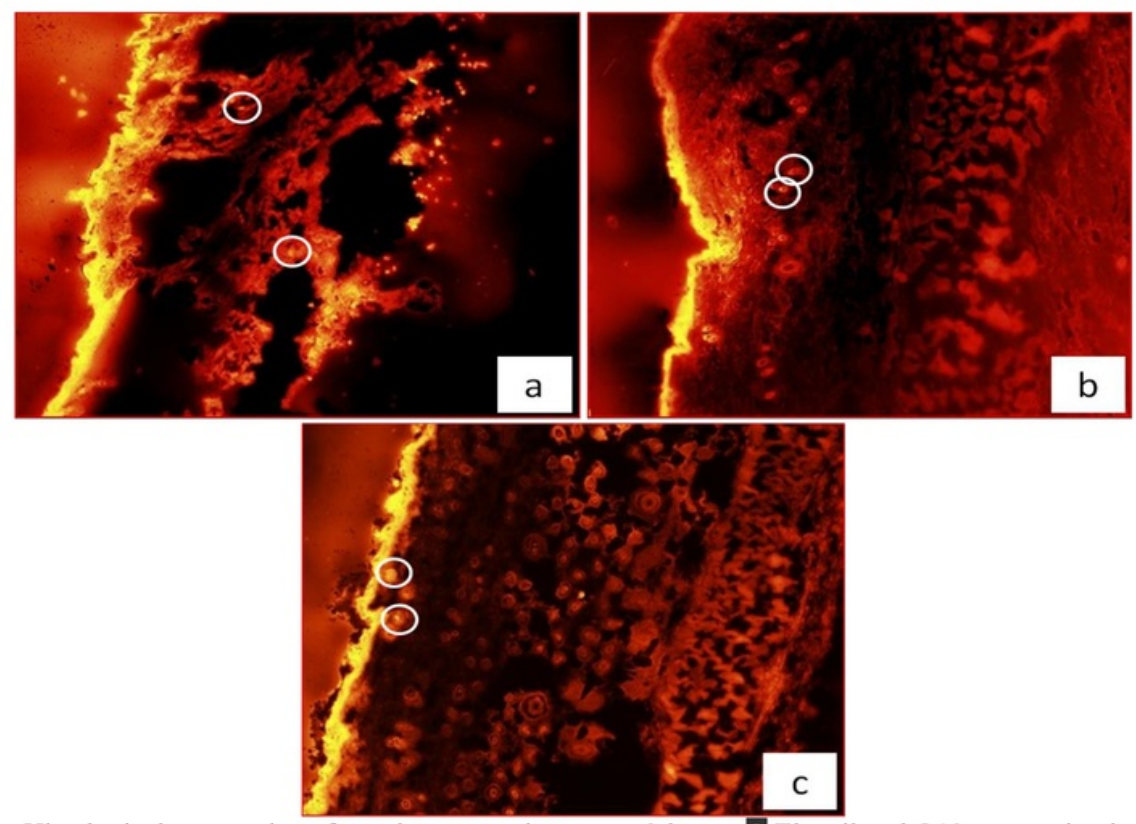

Figure 2: Histological preparations from the penetration test at 2 hours, $\mathrm{O}$ The oil and Q10 penetration in (a) blank nanoemulsion, (b) Q10 nanoemulsion, and (c) Q10 emulsion on the skin of Wistar rats was observed using a flourescence microscope (with ocular lens magnification $7.2 \mathrm{X}$ ).
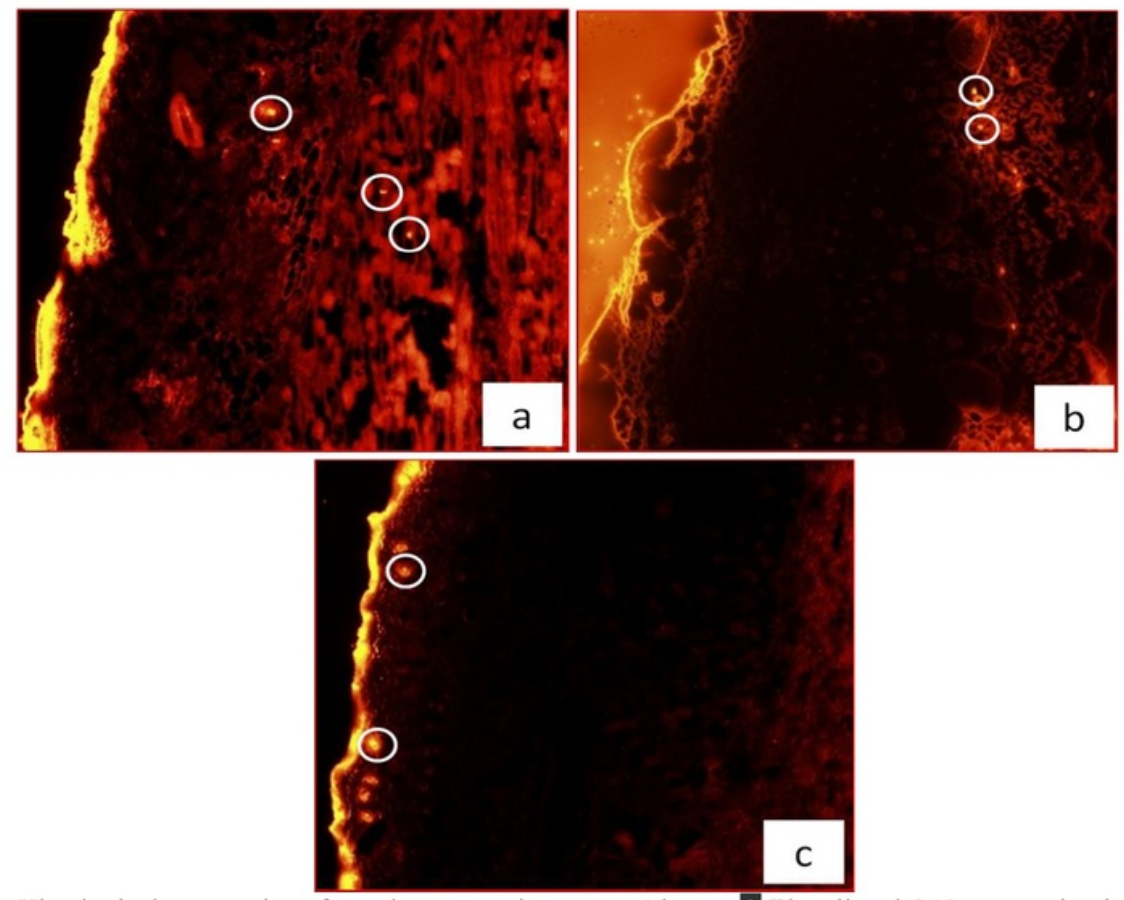

Figure 3: Histological preparations from the penetration test at 4 hours, $\mathrm{O}$ The oil and Q10 penetration in (a) blank nanoemulsion, (b) Q10 nanoemulsion, and (c) Q10 emulsion on the skin of Wistar rats was observed using a flourescence microscope (with ocular lens magnification $7.2 \mathrm{X}$ ). 
cosurfactants used for olive oil: Surfactants (Span 80 and Tween 80$)$ - cosurfactant $(96 \%$ ethanol): water phase (acetate buffer pH $4.2 \pm 0.5$ ) $=1: 9: 27.5$ with modifications during manufacture, since the type of oil used is different from previous research. The nanoemulsion was made by mixing $1 \%$ of Q10 and olive oil; it was stirred with a magnetic stirrer at a speed of 800 rpm for 5 minutes until it became homogeneous. Span 80 and Tween 80 were mixed and added to the mixture of Q10 and olive oil, then stirred with a magnetic stirrer with a speed of $800 \mathrm{rpm}$ for 5 minutes. The $96 \%$ ethanol was added into the mixture (Q10, olive oil, Tween 80 , and Span 80). Then, the mixture was stirred with a magnetic stirrer with a speed of $800 \mathrm{rpm}$ for 5 minutes. A $\mathrm{pH} 4.2 \pm 0.2$ acetate buffer solution is slowly added to a mixture for $50 \mathrm{ml}$. The mixture was then stirred again with magnetic stirrer for 10 minutes at a speed of 1000 $\mathrm{rpm}$, and then was sonicated for 10 minutes. Lastly, the mixture was shaken with a speed of $206 \mathrm{rpm}$ for 60 minutes to form a clear nanoemulsion.

Emulsion Preparation

In the preparation of emulsion, a preliminary experiment was carried out by a pseudo-ternary diagram. Q10 (1\%) was dissolved in olive oil $(35 \%)$ and then added with $22 \%$ of surfactant mixture (Tween 80 and Span 80 ), it was stirred until the mixture became homogeneous. Subsequently, a solution of acetate buffer $\mathrm{pH} 4.2 \pm 0.5$ (up to $100 \%$ ) was added into the mixture, and it was stirred until a homogeneous Q10 emulsion was obtained.
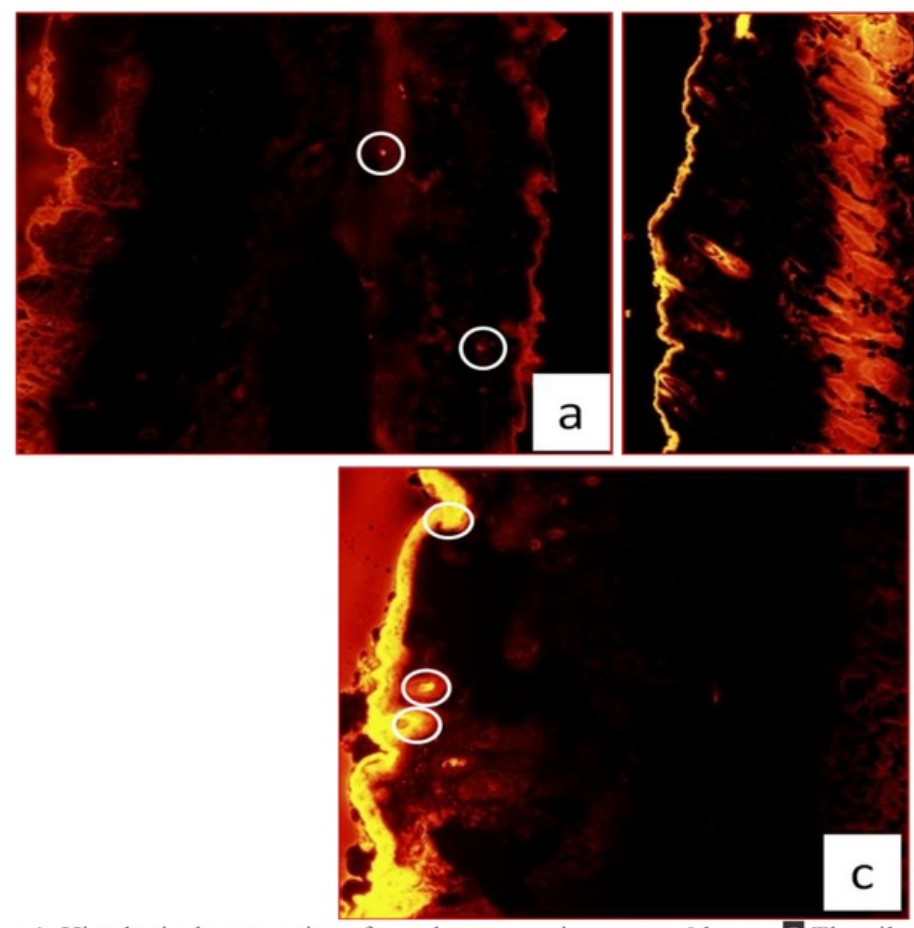

Figure 4: Histological preparations from the penetration test at 6 hours, $\mathrm{O}$ The oil and Q10 penetration in (a) blank nanoemulsion, (b) Q10 nanoemulsion, and (c) Q10 emulsion on the skin of Wistar rats was observed using a flourescence microscope (with ocular lens magnification 7.2 X).
The characterization Q10 nanoemulsion and Q10 emulsion include

Viscosity measurement using a cone and plate viscometer.

Droplet measurement of Q10 nano-emulsion by Delsa ${ }^{\mathrm{TM}}$ Submicron Nano Particle Size and Zeta Potential Dynamic Light Scattering tool. Meanwhile the Q10 emulsion droplet measurement by a light microscope.

The morphological observation of Q10 nanoemulsion droplet was observation by Transmission Electron Microscope (TEM) JEOL JEM 1400, while the Q10 emulsion droplet morphology observation by a light microscope.

Penetration Testing Using Fluorescent Microscope

Preparation of experimental animals

The number of animals as subjects in this research was calculated using the Federer equation: a number of 27 animals were divided into 9 groups of tests.

Group I, for treatment with blank nanoemulsion, Group II for treatment with Q10 nanoemulsion, and Group III for treatment with Q10 emulsion. The three groups were observed after 2 hours of application. Groups IV, V, and VI were observed after 4 hours of application. Groups VII, VIII, and IX were observed after 6 hours of application.

Preparation of rat membrane

The male Wistar rats were intramuscularly anesthetized with ketamine $(20 \mathrm{mg} / \mathrm{kg})$. The hair on the abdomen was shaved using a mechanical hair clipper. A number of 50

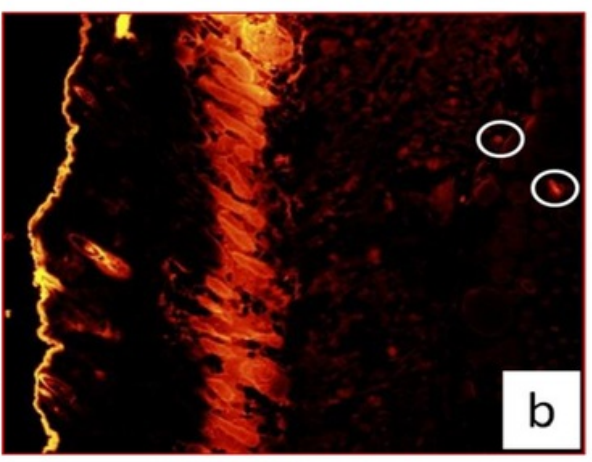


$\mathrm{mg}$ of the test sample was applied to the abdominal rat skin that had been removed of hair, with an area of $2 \times 3$ $\mathrm{cm}^{2}$. After a certain time $(2,4$, or 6 hours $)$, the rat were sacrificed by cervical dislocation. The skin that received treatments were prepared as histological sample and cut using frozen microtome. The sample preparations were observed with a fluorescence microscope.

\section{RESULTS AND DISCUSSION}

The empty (blank) nanoemulsion, Q10 nanoemulsion, and Q10 emulsion photos can be seen in Figure 1. The blank nanoemulsion appeared clear liquid. The addition of Q10 in nanoemulsion results in color changes into clear orange. Meanwhile, the resulting Q10 emulsion preparation is milky orange in color.

The results for $\mathrm{pH}$ observation found that the $\mathrm{pH}$ average for the three preparations were the same at $4.12 \pm 0.02$. The $\mathrm{pH}$ value was still in a vulnerable skin $\mathrm{pH}$ of 4.00 to 6.00. The morphological observation of the blank nanoemulsion droplet and Q10 nanoemulsion was performed with TEM (Transmission Electrone Microscopy), while the Q10 emulsion was performed using light microscopy. All three exhibit spherical morphology.

The measurement result of blank nanoemulsion droplet size and Q10 nanoemulsion using Delsa ${ }^{\mathrm{TM}}$ Submicron Nano Particle Size and Zeta Potential Dynamic Light Scattering identifies droplet sizes of $66.4 \pm 1.77 \mathrm{~nm}$ (blank nanoemulsion) and $70.07 \pm 12.42 \mathrm{~nm}$ (Q10 nanoemulsion). In the Q10 emulsion, measurement was conducted using a light microscope and the obtained droplet size is $21.063 \pm 3.57 \mu \mathrm{m}$. From the observation results of the three preparations droplet sizes, it were found that the droplet size of blank nanoemulsion $<$ Q10 nanoemulsi $<\mathrm{Q} 10$ emulsion. Droplet sizes can affect the speed of release and penetration of the drug. The smaller droplet size, will be increased the release and penetration rate $^{5}$. The observation results of polidispersity index of blank nanoemulsion and Q10 nanoemulsion preparation are $0.519 \pm 0.023$ and $0.489 \pm 0.018$, respectively. This indicates that the homogeneity of the droplet sizes of blank nanoemulsion and Q10 nanoemulsion are in the moderate category. From the viscosity test using a cone and plate viscometer, it was known that the viscosity of the blank nanoemulsion (7 CPa.s) $<$ Q10 nanoemulsi (10.5 CPa.s) $<$ Q10 emulsion (16 CPa.s).

The preparation with higher concentrations of oil produces higher viscosity. In emulsions, the amount of oil was $35 \%$ whereas in nanoemulsion the amount of oil was $2.66 \%$. Viscosity affects the release of the active ingredient; higher viscosity causes the release rate decreases.

The results of the penetration test using Wistar rats at 2 hours of treatment show that the penetration distances of blank nanoemulsion $(241.461 \pm 12.11 \mathrm{~m})$ and Q10 nanoemulsion $(312.034 \pm 31.273 \mathrm{~m})$ are far enough, both were reached the dermis. However, for the Q10 emulsion
$(25.625 \pm 2.653 \mathrm{~m})$, it was still on the epidermis. The observation of penetration distance at 4 hours after treatment show that penetration distance of blank nanoemulsion was $724.154 \pm 42.478 \mu \mathrm{m}$. For Q10 nanoemulsion, the distance was $755.724 \pm 38.744 \mu \mathrm{m}$, and for the Q10 emulsion the distance was $59.336 \pm$ $8.879 \mu \mathrm{m}$.

At the 4th hour, the Q10 emulsion penetration has also reached the dermis but the distance was still very close to the epidermis. At the 6 hours, the blank nanoemulsion penetration distance was $992.947 \pm 77.829 \mu \mathrm{m}$. For Q10 nanoemulsion the distance was $1024.405 \pm 108.534 \mu \mathrm{m}$, and for the Q10 emulsion the distance was $143.885 \pm$ $63.124 \mu \mathrm{m}$. The histological preparations of penetration test results on the skin of Wistar rats can be seen in Figures 2,3, and 4. Based on the data from penetration time and distance, a calculation of penetration rate of Q10 implies that the penetration rate of nanoemulsion (174.49 $\mu \mathrm{m} / \mathrm{h})>$ emulsion $(20.429 \mu \mathrm{m} / \mathrm{h})$. The increased penetration rate of Q10 in nanoemulsion can be caused due to the droplet size and viscosity of Q10 nanoemulsion that is smaller than that of the Q10 emulsion ${ }^{6}$.

\section{CONCLUSION}

From these results study it can be concluded that: Characteristics including droplet size and viscosity of Q10 nanoemulsion (70.07 $\pm 12.42 \mathrm{~nm}, 10.5$ CPa.s) smaller than Q10 emulsion $(21.063 \pm 3.57 \mu \mathrm{m}, 16 \mathrm{CPa} . \mathrm{s})$. The penetration rate of Q10 in nanoemulsion $(174.49 \mu \mathrm{m} / \mathrm{h})$ was higher than in the emulsion $(20.429$ $\mu \mathrm{m} / \mathrm{h})$.

\section{REFERENCES}

1. Angerhover, C. K., Maes, D., Giacomani, P. 2008. The Use of Natural Compounds and Botanicals in the Development of Anti Aging Skin Care Products. In Nava Dayan. Skin Aging Handbook. New York: William Andrew, pp. 205-264.

2. Gupta, A. and Kumar, R., 2010. Effect of geometry on droplet formation in the squeezing regime in a microfluidic T-junction. Microfluidics and Nanofluidics, 8(6), pp.799-812.

3. Rowe, R.C., Sheskey, P.J. and Quinn, M.E. eds., 2009. Handbook of pharmaceutical excipients (Vol.6). London: Pharmaceutical press.

4. Erawati T. Hendradi E., Soeratri W., 2014, Praformulation Study of p-Methoxycinnamic Acid (APMS) Nanoemulsion Using Vegetable Oils (Soybean Oil, Corn Oil, VCO), Int. J Pharm. Pharm. Sci, Vol. 6, Issue 2, pp 99-101.

5. Thakur, N., Garg, G., Sharma, P.K. and Kumar, N., 2012. Nanoemulsions: A Review on Various Pharmaceutical Application. Global J. Pharmacol, 6(3), pp. 222-225.

6. Tadros, T.F., 2005. Surfactants in Nano-Emulsions. Applied Surfactant s: Principles and Applications, $\quad$ pp. 285-308. 


\section{Penetration of Ubiquinone (Q10) Nanoemulsion Using Olive Oil Through Rat Skin}

ORIGINALITY REPORT

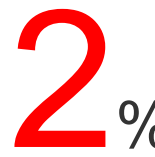

$\%$

$1 \%$

$1 \%$

$0 \%$

SIMILARITY INDEX

INTERNET SOURCES

PUBLICATIONS

STUDENT PAPERS

PRIMARY SOURCES

1 University of Tennessee, Knoxville

Publication

2 www.task39.org

Internet Source

\section{Exclude quotes}

Off

Exclude matches

Off

Exclude bibliography On 


\section{Penetration of Ubiquinone (Q10) Nanoemulsion Using Olive Oil Through Rat Skin}

GRADEMARK REPORT

FINAL GRADE

10

\section{PAGE 1}

PAGE 2

PAGE 3

PAGE 4
GENERAL COMMENTS

Instructor 